\title{
Avaliação do Google Sala de Aula como Ferramenta de Apoio ao Processo de Ensino-aprendizagem em um Curso de Licenciatura em Ciências Biológicas Presencial
}

\author{
Jonatas Martins - CERTEC / IFMA - jonmartins.martins@ gmail.com \\ Ariel Teles - IFMA - ariel.teles@ifma.edu.br \\ Davi Viana - UFMA - davi.viana@1sdi.ufma.br \\ Francisco José Silva - UFMA - fssilva@1sdi.ufma.br \\ Luciano Coutinho - UFMA - luciano.rc@ufma.br \\ Silmar Teixeira - UFPI - silmarteixeira@ufpi.edu.br
}

Resumo. Este artigo apresenta uma investigação sobre a usabilidade do Ambiente Virtual de Aprendizagem (AVA) Google Sala de Aula, visando identificar quais contribuições e melhorias a sua utilização pode trazer para o processo de ensino-aprendizagem. Foi aplicado um questionário, com a participação de 28 alunos em um curso de Licenciatura em Ciências Biológicas. Os resultados demonstram uma maior flexibilidade de acesso pelos alunos às atividades realizadas por meio do AVA e também com o uso de dispositivos móveis, tanto dentro quanto fora da sala de aula. Dessa forma, os resultados sugerem que a ferramenta é de fácil utilização, proporcionando maior interatividade e colaboração através da mediação docente.

Palavras-chave: Usabilidade; Google Sala de Aula; Processo de Ensino-aprendizagem.

\section{Google Classroom Assessment as a Tool to support the Teaching-learning Process at a Face-to-face Biological Licentiate Brazilian Course}

\begin{abstract}
This paper presents a usability investigation of the Virtual Learning Environment (VLE) named Google Classroom, seeking to identify what contributions and improvements its use brings for the teaching-learning process. An online questionnaire was applied, with the participation of 28 students at a face-to-face Biological Licentiate Brazilian course, Results show greater flexibility of access by the respondents to the activities performed through the VLE and also with the use of mobile devices, both inside and outside the classroom. Therefore, the results suggest that Google Classroom is a user-friendly tool, providing greater interactivity and collaboration through teacher mediation.
\end{abstract}

Keywords: Usability; Google Classroom; Teaching-learning Process.

\section{Introdução}

A tecnologia facilita as atividades diárias dos professores e seus alunos. O uso de dispositivos móveis pelas instituições de ensino é uma prática que surgiu ao longo das últimas décadas, impactando a educação, gerando novas formas de acessar, transmitir, e obter novos saberes. Isso faz as Tecnologias de Informação e Comunicação (TICs) ferramentas imprescindíveis na prática educativa e na edificação do conhecimento (Camacho, 2017). O uso adequado das TICs pelas instituições de ensino amplia as habilidades, métodos e processos de obtenção de conhecimento de seus usuários a partir da introdução de ferramentas que os auxiliem. Esse auxílio aos educadores e educandos pode ser realizado através de diversos dispositivos, tais como smartphones, tablets e notebooks, os quais normalmente passam a dividir o espaço em 
sala de aula com demais objetos tradicionais, como o quadro negro e livros escolares (Martins \& Maschio, 2014).

Os Ambientes Virtuais de Aprendizagem (AVAs) são "plataformas que disponibilizam funcionalidades para o auxílio no aprendizado online, seja na modalidade à distância ou como apoio ao ensino presencial" (Souza \& Souza, 2016). Diante desse contexto, com o avanço da tecnologia e expansão da Internet, inclusive na área de educação, o uso de recursos digitais através de ferramentas de AVA como uma forma de complementar aos estudos vem sendo investigado por diversos pesquisadores (Lima \& Silva, 2017). Em particular, o Google Sala de Aula ${ }^{1}$ (ou Google Classroom) é uma plataforma de AVA social, gratuita e, segundo a empresa Google (2014, p.2), "possibilita a criação e organização rápida de tarefas, envio eficiente de comentários e a fácil comunicação com os alunos". Como uma sala de aula virtual, ela permite, entre outras funcionalidades, o compartilhamento de conteúdo, a discussão de textos, imagens, áudios e vídeos através de outros recursos como dispositivos móveis ou aplicativos Google como o Gmail e Google Drive, ferramentas integradas e que funcionam em conjunto com o Google Sala de Aula (Araújo, 2016).

O objetivo desta pesquisa foi verificar quais contribuições a utilização do AVA Google Sala de Aula traz ao processo de ensino-aprendizagem de um grupo de alunos de Ciências Biológicas. Para isso, analisou-se a percepção dos alunos quanto à inserção do AVA em suas atividades em um curso presencial. Dessa forma, buscou-se identificar a eficácia desse recurso no ensino híbrido (Silva \& Santos Júnior, 2019).

O presente artigo é dividido em 5 seções, incluindo a introdução. A Seção 2 discute os trabalhos relacionados. Na Seção 3, é detalhada a metodologia adotada para a condução desta pesquisa. A Seção 4 apresenta os resultados e discussão da pesquisa. Por fim, na Seção 5, descreve-se a conclusão do trabalho.

\section{Trabalhos Relacionados}

Alguns estudos na literatura têm investigado os AVAs (Mueller \& Strohmeier, 2011) e, mais especificamente, o Google Sala de Aula (Kumar \& Bervell, 2019). Por exemplo, Souza \& Souza (2016) apresentaram um experimento utilizando o Google Sala de Aula em uma turma de ensino médio da disciplina de Matemática, analisando o conceito de Aprendizagem Colaborativa. Os autores constataram que a inclusão dessa ferramenta desperta um maior interesse dos alunos, bem como favorece trabalhos que incentivam uma maior interação e colaboração entre eles.

Lima \& Silva (2017) relataram uma experiência de avaliação do Google Sala de Aula em cinco disciplinas de cursos técnicos e superiores na área de informática. Eles observaram que a maioria dos alunos obteve uma maior interação com o professor, além de uma melhoria na pontualidade e entrega de trabalhos. Um resultado semelhante foi identificado por Silva \& Netto (2018) durante a realização de um experimento com alunos do $3^{\circ}$ ano do Ensino Médio em uma disciplina de Química Orgânica e utilizando o Google Sala de Aula. Estes autores obtiveram dados que consideraram didática a comunicação por meio da inserção da plataforma, além de incentivar os alunos a serem mais efetivos na condução de suas atividades durante as aulas.

Pedroso (2018) buscou investigar se o Google Sala de Aula proporciona uma melhor otimização do tempo e quais benefícios de sua implantação em sala de aula. Para isso, realizou um experimento com o professor e alunos da disciplina de Montagem

\footnotetext{
${ }^{1}$ https://classroom.google.com

V. $17 \mathrm{~N}^{\mathrm{o}} 3$, dezembro, 2019

DOI:
} RENOTE 
e Manutenção de um Curso Técnico em Informática. Neste experimento se confirmou, através da aplicação de um questionário, a importância desse recurso. Schiehl e Gasparini (2016) apresentaram uma proposta de ensino híbrido pelo modelo de rotação por estações, utilizando o Google Sala de Aula. Eles observaram um maior contato entre professor e estudante durante uma experiência realizada em aulas de Matemática do $2^{\circ}$ ano no ensino médio. Esse contato possibilitou um maior engajamento dos estudantes durante as aulas e atitudes mais colaborativas.

A pesquisa descrita neste presente artigo apresenta resultados positivos de forma semelhante aos trabalhos relacionados descritos acima, como maior agilidade na troca de informações, interatividade, colaboração e, conforme Pedroso (2018, p. 17), uso de "mediação do conhecimento e métodos avaliativos" com os estudantes. Resultados que confirmam os benefícios esperados pela utilização do AVA. Nos resultados negativos demonstrados nos trabalhos relacionados, alguns apresentaram acesso limitado ou fragilidade em relação à velocidade de navegação (Silva \& Netto, 2018), outros, resistência por parte de alguns alunos na utilização do AVA (Lima \& Silva, 2017). Situações que não ocorreram na atual pesquisa, pois o acesso à Internet poderia ser feito tanto através dos próprios dispositivos móveis dos participantes quanto no laboratório de informática da instituição de ensino. Além disso, os participantes não demonstraram resistência, uma vez que a maioria tinha conhecimento prévio em utilizar o sistema, conforme resultados do instrumento de pesquisa.

\section{Metodologia}

Este artigo descreve uma experiência que avaliou a utilização do AVA Google Sala de Aula. Fizeram parte deste estudo 28 alunos do $4^{\circ}$ período do Curso Superior de Licenciatura em Ciências Biológicas na disciplina de Informática na Educação. O curso é ofertado pelo Instituto Federal do Maranhão - Campus Barreirinhas.

\subsection{Etapas da pesquisa}

O trabalho foi dividido em quatro etapas apresentadas na Tabela 1. A duração das etapas 1 e 2 foi de 4 meses, de março a junho de 2019. A realização da etapa 3 foi no dia 03 de junho de 2019. Já a aplicação do questionário iniciou no dia 18 de junho e finalizou no dia 26 de junho de 2019.

Tabela 1 - Etapas da Pesquisa com o uso do AVA Google Sala de Aula.

\begin{tabular}{|c|l|}
\hline ETAPAS & \multicolumn{1}{|c|}{ APRESENTAÇÃO PARA O DESENVOLVIMENTO PRÁTICO } \\
\hline $1^{\text {a }}$ & $\begin{array}{l}\text { Orientados pelo professor, os alunos através do AVA obtiveram acesso aos } \\
\text { conteúdos ministrados em sala de aula, os quais incluíram: slides e apostilas } \\
\text { criados pelo professor e disponibilizados em formato PDF, videoaulas criadas pelo } \\
\text { professor para visualização online e também para download, e vídeos de acesso } \\
\text { público da plataforma YouTube. Os conteúdos de autoria do professor foram } \\
\text { disponibilizados através do Google Drive (ferramenta integrada ao Google Sala de } \\
\text { Aula). A organização dos conteúdos inseridos no AVA seguiu a sequência da } \\
\text { ementa do Plano de Ensino do professor. }\end{array}$ \\
\hline $2^{\text {a }}$ & $\begin{array}{l}\text { O professor disponibilizou atividades avaliativas, leitura e elaboração de artigos } \\
\text { científicos para os alunos desenvolverem através do AVA. Para a realização de } \\
\text { atividades avaliativas, as quais abordaram o conteúdo da disciplina, o professor }\end{array}$ \\
\hline
\end{tabular}

V. $17 \mathrm{~N}^{\mathrm{o}}$ 3, dezembro, 2019 RENOTE DOI: 


\begin{tabular}{|c|l|}
\hline & $\begin{array}{l}\text { utilizou o Google Forms (ferramenta integrada ao Google Sala de Aula). Os } \\
\text { artigos para leitura foram disponibilizados para visualização online e download } \\
\text { através do Google Drive. Além disso, o professor indicou que o artigo científice } \\
\text { deveria ser desenvolvido de forma colaborativa no Google Docs (ferramenta } \\
\text { integrada ao Google Sala de Aula) e compartilhado com ele, de maneira a permitiı } \\
\text { o acompanhamento e orientação da escrita do trabalho. }\end{array}$ \\
\hline $3^{\text {a }}$ & $\begin{array}{l}\text { Realizou-se uma visita à instituição de educação para a apresentação da proposta } \\
\text { de estudo de percepção dos alunos e sobre a avaliação da ferramenta em face do } \\
\text { desenvolvimento das atividades realizadas na disciplina de Informática no } \\
\text { Educação. Este momento serviu também para que os alunos que aceitaram } \\
\text { participar da pesquisa pudessem assinar o Termo de Consentimento Livre e } \\
\text { Esclarecido. }\end{array}$ \\
\hline $4^{\text {a }}$ & $\begin{array}{l}\text { Um questionário online foi aplicado para coleta de opinião e dados de cada aluno } \\
\text { participante. }\end{array}$ \\
\hline
\end{tabular}

\subsection{Coleta de dados}

Para se alcançar os resultados abordados nesta pesquisa, foi disponibilizado um questionário via plataforma Google Forms, composto de quatro partes. A primeira parte continha questões relacionadas ao perfil do participante. A segunda parte estava relacionada a usabilidade do sistema. A terceira parte buscava informações sobre o uso do AVA em atividades dentro e fora da sala de aula. Por fim, a quarta parte abordou questões relacionadas a futura vida profissional docente dos participantes.

Para avaliar a usabilidade do AVA Google Sala de Aula, foi utilizado o System Usability Scale (SUS), o qual consiste de um questionário simples e de rápida aplicação, por onde é determinado a satisfação do usuário em relação ao sistema e seu uso. As questões do SUS são enumeradas abaixo. As respostas do questionário SUS são coletadas para medir a avaliação de usabilidade e satisfação do usuário sobre um sistema computacional (e.g., programa de computador, aplicativos móveis, sites web), usando a escala Likert (Likert, 1932). Nesta escala, a pontuação é dada de 1 a 5, com as seguintes possíveis respostas: 1 (Discordo Plenamente - DP), 2 (Discordo - D), 3 (Neutro - N), 4 (Concordo - C) e 5 (Concordo Plenamente - CP). As questões utilizadas foram:

1. Eu acho que gostaria de usar o Google Sala de Aula com frequência.

2. Eu acho o Google Sala de Aula desnecessariamente complexo.

3. Eu achei o Google Sala de Aula fácil de usar.

4. Eu acho que precisaria de ajuda de uma pessoa com conhecimentos técnicos para usar o Google Sala de Aula.

5. Eu acho que as várias funções do Google Sala de Aula estão muito bem integradas.

6. Eu acho que o Google Sala de Aula apresenta muita inconsistência.

7. Eu acho que as pessoas podem aprender como usar o Google Sala de Aula rapidamente.

8. Eu achei o Google Sala de Aula complicado de usar.

9. Eu me senti confiante ao usar o Google Sala de Aula.

10. Eu precisei aprender várias coisas novas antes de conseguir usar o Google Sala de Aula.

Para se chegar à pontuação final do SUS, deve-se seguir os seguintes procedimentos: nas questões ímpares $(1,3,5,7$ e 9), a pontuação corresponde a posição 
na escala menos 1 , ou seja, subtrai-se um; nas questões pares $(2,4,6,8$ e 10$)$, subtrai-se 5 pelo valor posicionado na escala. Em seguida soma-se as pontuações de cada item, multiplicando a soma das pontuações por 2,5. O resultado corresponde ao valor global entre 0 e 100 da escala de usabilidade (Brooke, 1996). Na terceira parte do questionário, foi feita uma análise de avaliação do uso do Google Sala de Aula em atividades dentro ou fora do ambiente escolar. Seguindo o mesmo modelo de questionamento da escala Likert, em que foi abordado através de cinco questões objetivas, sua interação e otimização, entendimento e manuseio, acessibilidade e flexibilidade, desempenho e organização dos conteúdos, além de sua praticidade. Por fim, a quarta parte, com quatro questões objetivas, sendo uma questão utilizando a escala Likert e as demais em que o respondente optaria entre "Sim" ou "Não". As questões tinham por base a experiência de vida acadêmica observada e adquirida por todos os participantes. Procurou-se conhecer se estes estariam dispostos após sua futura formação docente a dar continuidade em seus ensinamentos para uso próprio nas instituições, da aplicação dessa plataforma, utilizando-a como uma ferramenta de apoio em sala de aula e se recomendariam sua utilização.

\section{Resultados e Discussão}

A primeira parte do instrumento de pesquisa teve como objetivo levantar informações sobre o perfil dos alunos participantes do curso de Licenciatura em Ciências Biológicas, e obtiveram-se os seguintes resultados: o questionário foi respondido por 28 participantes com faixa etária entre 18 e 44 anos, sendo que a maioria dos participantes, 12 (43\%), se encontram entre 18 e 21 anos; em relação ao gênero dos participantes, a maioria, 15 (54\%), foram mulheres.

Quando indagados sobre qual dos dispositivos tecnológicos eles usavam para acessar a Internet e, consequentemente, o AVA para seus estudos, a maioria dos participantes, $23(82 \%)$, relataram que faziam uso do smartphone (Figura 1).

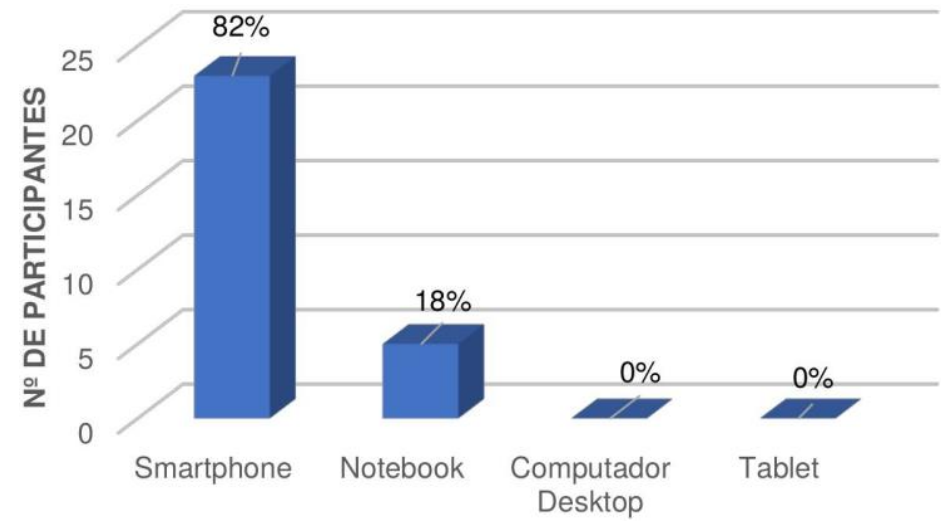

Figura 1 - Dispositivos computacionais utilizados.

Essa preferência pelo smartphone, como ferramenta de auxílio nos estudos, é também observada na pesquisa de Lopes (2016, p. 01), ao "verificar a utilização de TICs como ferramentas auxiliares no ensino de Biologia em escola da região metropolitana de Porto Alegre e a integração destes atores educacionais no ciberespaço" em que metade dos seus participantes também fizeram uso do smartphone.

Esse elevado número de usuários de smartphone pode estar relacionado a um custo acessível e sua popularidade, uma vez que esse dispositivo, atualmente, faz parte 
da vida diária de alunos nas instituições de ensino, como também fora delas (Pereira, 2016). Nesse contexto, a maioria dos participantes (82\%) também relataram que o local onde mais utilizavam a Internet para estudos é em sua própria casa, como pode ser visto na Figura 2.

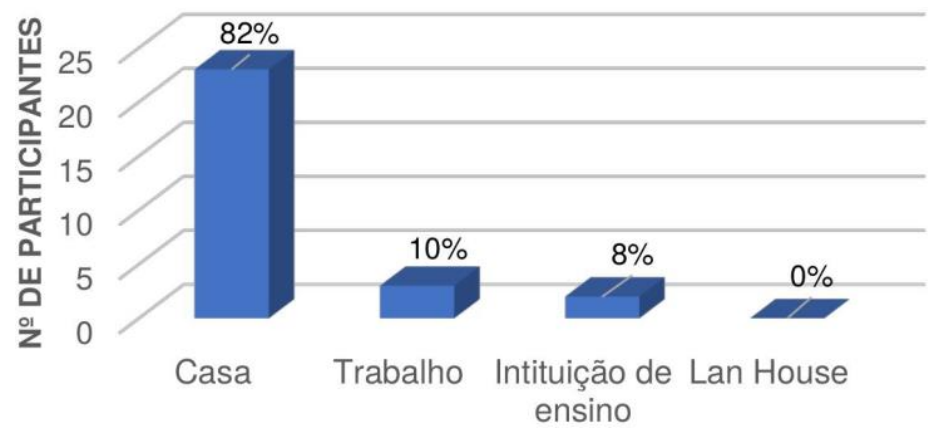

Figura 2 - Lugar mais utilizado para estudos.

A Figura 2 demonstra a importância da criação ou escolha adequada de uma sala de aula virtual. É necessário ter acesso através de ferramentas com conexão à Internet de forma presencial, como fora de seus limites, considerando o auxílio da Internet como principal ferramenta integradora de professores e alunos fora da sala de aula (Filho e Saeger, 2016).

\subsection{Análise da avaliação de usabilidade}

A Figura 3 apresenta a avaliação da usabilidade de acordo com o SUS, a qual resultou na pontuação média geral de 85,89 , desvio padrão de 10,7 , valor mínimo de 52,5 e máximo de 100,0; o qual considera o sistema "excelente" em termos de usabilidade (Bangor et al., 2009).

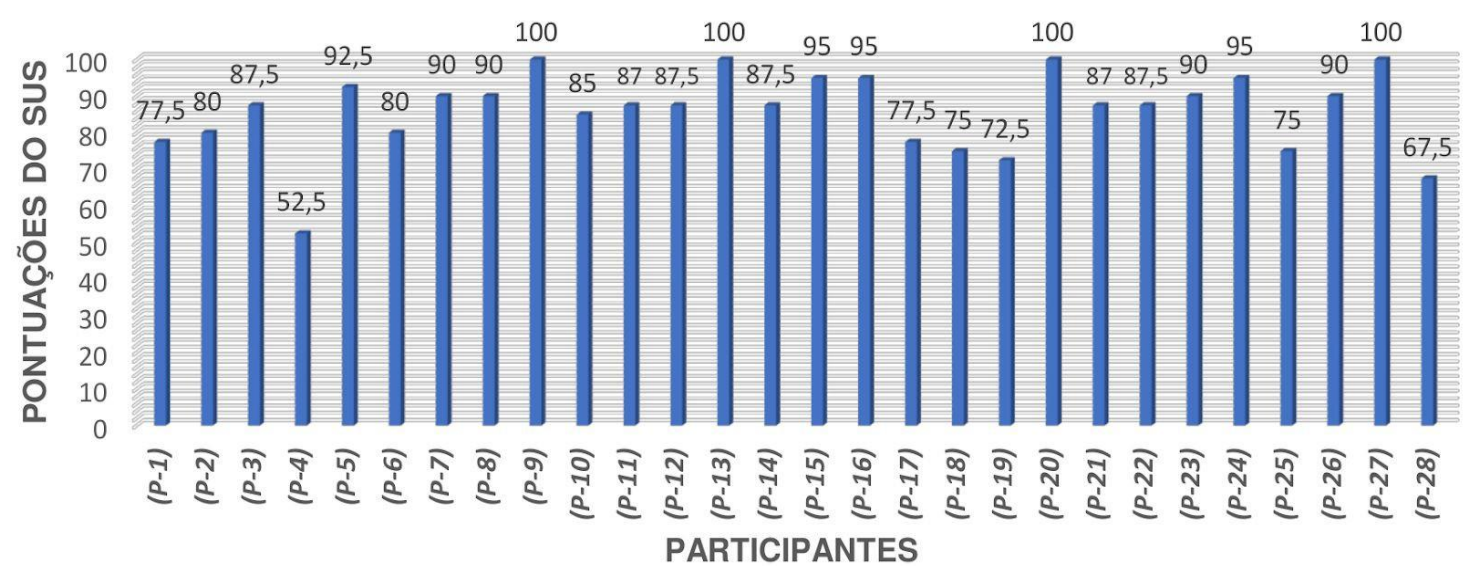

Figura 3 - Pontuação do SUS por participante.

O resultado do SUS é apresentado em detalhes na Tabela 2, o qual demonstra que a maioria dos participantes expressou respostas positivas a respeito da "Frequência de uso" (90\%), da "Facilidade ao usar o sistema" (93\%), das "Funções integradas" $(90 \%)$, na "Aprendizagem rápida" (82\%) e na "Confiança para usar o sistema" (75\%). Apenas um sujeito discordou quanto à "Aprendizagem rápida" e "Confiança para usar o sistema". Ainda como respostas positivas, a maioria dos participantes foi discordante V. $17 \mathrm{~N}^{\circ}$ 3, dezembro, 2019 RENOTE DOI: 
em relação a "Complexidade" (68\%) do sistema, a sua "Inconsistência" (93\%), o "Auxílio para usar o Google Sala de Aula" (86\%), se o "Sistema é complicado" (82\%) e em "Ter conhecimento prévio para utilizar o sistema" (82\%).

Tabela 2 - Resultado de avaliação do sistema no SUS.

\begin{tabular}{|c|c|c|c|c|c|}
\hline Questões & $\mathbf{D P}$ & $\mathbf{D}$ & $\mathbf{N}$ & $\mathbf{C}$ & $\mathbf{C P}$ \\
\hline $\mathbf{1}$ & 0 & 0 & 3 & 7 & 18 \\
\hline $\mathbf{2}$ & 18 & 1 & 6 & 2 & 1 \\
\hline $\mathbf{3}$ & 0 & 0 & 2 & 5 & 21 \\
\hline $\mathbf{4}$ & 18 & 6 & 1 & 1 & 2 \\
\hline $\mathbf{5}$ & 0 & 0 & 3 & 9 & 16 \\
\hline $\mathbf{6}$ & 19 & 7 & 2 & 0 & 0 \\
\hline $\mathbf{7}$ & 0 & 1 & 4 & 8 & 15 \\
\hline $\mathbf{8}$ & 20 & 3 & 3 & 1 & 1 \\
\hline $\mathbf{9}$ & 1 & 0 & 6 & 8 & 13 \\
\hline $\mathbf{1 0}$ & 20 & 3 & 3 & 0 & 2 \\
\hline
\end{tabular}

\subsection{Análise da avaliação sobre atividades dentro e fora da sala de aula}

A Tabela 3 apresenta os resultados sobre a avaliação do uso do Google Sala de Aula em atividades dentro ou fora do ambiente escolar. Com esses resultados, é possível verificar o desempenho e flexibilidade do AVA face ao envolvimento e interação dos participantes com suas atividades. Como visto, a maioria dos participantes expressou respostas positivas: $\mathrm{C}$ e $\mathrm{CP}$ nas quatro primeiras questões e $\mathrm{DP}$ e $\mathrm{D}$ na última, uma vez que esta é uma afirmação negativa.

Tabela 3 - Uso do Google Sala de Aula em atividades dentro e fora da sala de aula.

\begin{tabular}{|l|c|c|c|c|c|}
\hline \multicolumn{1}{|c|}{ Questões } & DP & D & N & C & CP \\
\hline $\begin{array}{l}\text { O Google Sala de Aula otimiza o tempo, me sinto conectado } \\
\text { com a turma e o professor, interagindo sobre os assuntos das } \\
\text { aulas. }\end{array}$ & 0 & 0 & 5 & 7 & 16 \\
\hline $\begin{array}{l}\text { O Google Sala de Aula é de rápido entendimento e manuseio } \\
\text { na realização e entrega de atividades. }\end{array}$ & 0 & 0 & 1 & 9 & 18 \\
\hline $\begin{array}{l}\text { O Google Sala de Aula me permite acessar os conteúdos das } \\
\text { aulas através de diferentes dispositivos. }\end{array}$ & 0 & 0 & 1 & 3 & 24 \\
\hline $\begin{array}{l}\text { O Google Sala de Aula é mais fácil e rápido para rever } \\
\text { atividades anteriores e organizar os conteúdos que são dados } \\
\text { em aula. }\end{array}$ & 0 & 0 & 3 & 13 & 12 \\
\hline O Google Sala de Aula é uma plataforma lenta para navegação. & 20 & 6 & 1 & 1 & 0 \\
\hline
\end{tabular}

\subsection{Opinião dos participantes sobre futuras atuações com o uso do AVA}

A última parte do questionário abordou questões referentes à qualidade de futura formação docente dos participantes, em que a maioria (70\%) concordou que o uso do Google Sala de Aula ajuda a melhorar ainda mais o processo de ensino-aprendizagem, V. $17 \mathrm{~N}^{\circ}$ 3, dezembro, 2019 RENOTE DOI: 
tanto em aulas de Ciências/Biologia quanto em outras disciplinas. O trabalho de Silva \& Netto (2018, p.124), em uma disciplina de química, também demonstrou essa variedade e melhora no ensino com o uso do AVA, em que os resultados foram de que o "o caráter didático e interativo na plataforma favorece a aprendizagem dinâmica. $\mathrm{O}$ uso da plataforma estreitou as relações entre os alunos, mantendo-os mais tempo online e atentos aos avisos/comentários inseridos na mesma".

Outras duas indagações, a primeira com o seguinte enunciado "Considerando sua futura vida profissional (docente), você utilizaria o Google Classroom?" e outra se "Você recomendaria o uso do Google Classroom na(s) instituição(s) de ensino em que irá lecionar?" tiveram todas as respostas "Sim". Silva \& Netto $(2018$, p.127) relataram que uma colega professora, por meio de observações na pesquisa realizada, se mostrou "satisfeita com os resultados, sendo que também começou a usar a plataforma para fins educacionais". Na pesquisa de Pedroso (2018, p.27), é descrito um relato de um professor que utiliza o Google Sala de Aula "por ser uma ferramenta gratuita, de fácil acesso, além de ser uma excelente forma de incentivar os alunos a pesquisarem e se envolverem mais com a disciplina fora do ambiente escolar", mostrando-se engajado em manter o uso do AVA "como ferramenta de ensino e engajado em conscientizar o corpo docente de sua importância no ensino". Todas as respostas relatadas pelos autores da literatura demonstram resultados similares aos encontrados na presente pesquisa.

A última questão teve o seguinte enunciado "Você acha que seus futuros alunos aprenderiam como usar o Google Classroom facilmente?", em que 27 participantes $(96 \%)$ confirmaram com "Sim" essa indagação. Esse resultado está de acordo com a questão 7 do SUS, em que a maioria disse que pensa que as pessoas podem aprender como usar o Google Sala de Aula rapidamente. Em ambas questões apenas um discente não concordou com as afirmações positivas nas duas questões levantadas.

\subsection{Lições aprendidas}

$\mathrm{Na}$ atual geração de jovens estudantes, a qual vivenciamos, os alunos já conseguem facilmente entender e manipular muitos dos novos recursos tecnológicos. Com os estudantes participantes desta pesquisa não foi diferente. Através do Google Sala de Aula, eles tiveram a possibilidade de aliar a tecnologia aos estudos, e o professor participante, como mediador, pôde contribuir para que os alunos pudessem ter uma aprendizagem significativa.

Percebeu-se então, com o experimento realizado, que o uso do AVA Google Sala de Aula diminuiu custos de execução da disciplina (e.g., por não utilizar meio impresso de disponibilização de conteúdos) e otimizou o tempo, auxiliando na melhoria da condução das aulas do curso de Informática na Educação. Dessa forma, acredita-se que o uso de AVAs por instituições de ensino pode ser vantajoso aos usuários (i.e., professores e alunos), mesmo em cursos realizados em sua totalidade de carga-horária na modalidade presencial.

\section{Conclusão}

Este trabalho objetivou identificar as contribuições que o uso do AVA Google Sala de Aula traria para o processo de ensino-aprendizagem de estudantes de uma disciplina de Informática na Educação de um curso de Licenciatura em Ciências Biológicas com carga horária totalmente presencial. Realizou-se um experimento em que os resultados apontaram uma boa percepção dos participantes em relação à inserção 
e eficácia desta plataforma uma vez que eles relataram experiências positivas sobre a usabilidade do AVA. Dessa forma, os resultados da pesquisa sugeriram que o AVA se mostrou uma ferramenta com potencial para melhoria do ensino, pois proporcionou uma aprendizagem mais ativa ao criar novas possibilidades de obtenção de conhecimento (e.g., com o uso de dispositivos móveis para acessar o AVA) e durante a comunicação dos participantes, fazendo-os trabalhar de maneira colaborativa e tornando-os mais independentes em seus estudos. Adicionalmente, os resultados indicaram fortemente a adesão dos futuros professores licenciados em Biologia pela utilização de AVAs. Portanto, com a realização de um experimento e dos resultados obtidos, acredita-se que o objetivo geral desta pesquisa foi alcançado.

Planos para trabalhos futuros incluem a realização de experimentos em disciplinas com conteúdos não relacionados à informática, uma vez que o fato dos estudantes, e também o professor, estarem inseridos em uma disciplina com conteúdos sobre tecnologia (i.e., Informática na Educação) favoreceu a obtenção de resultados favoráveis ao uso da ferramenta proposta. Uma possibilidade de trabalho a ser realizado pode ser a condução de pesquisa similar em uma disciplina técnica do curso. Outra possível investigação a ser realizada futuramente é a realização de experimentos com outros AVAs visando verificar se os resultados serão também promissores comparando-os com aqueles obtidos e relatados neste trabalho.

\section{Agradecimentos}

Os autores agradecem o Centro de Referência Tecnológica (CERTEC) do Instituto Federal de Educação, Ciência e Tecnologia do Maranhão pelo apoio dado para a realização desta pesquisa.

\section{Referências Bibliográficas}

ARAÚJO, H. O uso das ferramentas do aplicativo "Google sala de aula" no ensino de matemática. Catalão-GO: Mestrado Profissional em Matemática em Rede Nacional/UFG, 2016. 93p. Dissertação de Mestrado.

BANGOR, A.; KORTUM, P.; MILLER, J. Determining what individual SUS scores mean: Adding an adjective rating scale. Journal of Usability Studies. v. 4, n. 3, p. 114-123, 2009.

BROOKE, J. SUS: A Quick and Dirty Usability Scale. In Jordan, P. W., Thomas, B. A. Weerdmeester and McClelland, I. (Ed). Usability evaluation in industry. London: Taylor \& Francis, 1996. p. 189-194.

CAMACHO, C. Recursos Tecnológicos e Motivação para a Aprendizagem. Porto-PT: Universidade Fernando Pessoa, 2017. 121p. Dissertação de Mestrado.

FILHO, A.; SAEGER, M. Contribuições das tecnologias da informação e comunicação para a aprendizagem em Ciências Contábeis. Revista Tecnologia na Educação. Editora da UTFPR. v. 8, n. 14, p. 1-11, 2016.

GOOGLE. Google Apps: O que há de novo. Disponível em: 〈https://goo.gl/MFv9PW〉. Acesso em: 20 set. 2019. 2014. 
KUMAR, J.A.; BERVELL, B. Google Classroom for mobile learning in higher education: Modelling the initial perceptions of students. Education and Information Technologies. Springer. v. 24, n. 2, pp 1793-1817, 2019.

LIMA, D.A.; ZATI, A.F.; SILVA, E. C. Análise de dados no Google Classroom para auxiliar na diminuição do distanciamento transacional nas disciplinas da área de informática. In: TISE Conferência Internacional sobre Informática na Educação, 13., 2017, Fortaleza. Anais. Santiago de Chile.

LIKERT, R. A Technique for the Measurement of Attitudes. Archives of Psychology. v. 22, n. 140, 1932, 55 p.

LOPES, L.A. Olhar digital na escola: a cibercultura nas aulas de Biologia em uma escola da periferia de Canoas, RS. Revista Tecnologia na Educação. Editora da UTFPR. v. 8, n. 14, p. 1- 12, 2016.

MARTINS, O.B.; MASCHIO, E.C.F. As Tecnologias Digitais na Escola e a Formação Docente: representações, apropriações e práticas. Actualidades Investigativas en Educación. Editora da Universidad de Costa Rica. v. 14, n.3, p.1-21, 2014.

MUELLER, D.; STROHMWIER, S. Design characteristics of virtual learning environments: state of research. Computers \& Education. Elsevier. v. 57, n. 4, p. 2505-2516, 2011.

PEDROSO, C.V. O uso do google classroom como ambiente virtual de aprendizagem em um curso técnico de informática. Santana do Livramento-RS: Curso de Especialização em Tecnologias da Informação e da Comunicação Aplicadas à Educação/UFSM, 2018. 30p. Artigo de Especialização.

PEREIRA, J.S. Do consumo as apropriações: o uso de smartphones por estudantes do ensino médio em Cuiabá. Anagrama. v. 10, n. 1, p. 1-19, 2016.

SCHIEHL, E.P.; Gasparini, I. Contribuições do Google Sala de Aula para o Ensino Híbrido. RENOTE. UFRGS. v. 14, n. 2, 2016.

SILVA, G.; NETTO, J.F. Um Relato de Experiência Usando Google Sala de Aula para Apoio à Aprendizagem de Química. In: Anais do Workshop de Informática na Escola, 24., 2018, Fortaleza.

SILVA, S.S.; SANTOS JÚNIOR, A.C.P. Google Sala de Aula como Ambiente Virtual de Aprendizagem no Ensino Superior Híbrido: Uma Revisão da Literatura. EaD em Foco. Fundação Cecierj. v. 9, n. 1, 2019.

SOUZA, A.; SOUZA, F. Uso da Plataforma Google Classroom como ferramenta de apoio ao processo de ensino e aprendizagem: Relato de aplicação no ensino médio. Rio Tinto, PB: Licenciatura em Ciência da Computação/UFPB, 2016. 27p. TCC. 